\title{
Transport of Critically Ill Patients - A Review of Early Interventions, Protocols, and Recommendations
}

\author{
Sibghatullah M Khan', Marcus D. Lance ${ }^{2}$, Mariam Ali Karrar Elobied ${ }^{3}$ \\ ${ }^{1,3}$ Associate Consultant, Anesthesia, ICU \& Perioperative Medicine, Hamad Medical Corporation, Qatar. \\ ${ }^{2}$ Senior Consultant Anesthesia \& ICU, Voice Chair, Head of Research. Department of Anesthesia, ICU \& \\ Perioperative Medicine, Hamad Medical Corporation, Qatar.
}

Corresponding Author: Sibghatullah M Khan

\begin{abstract}
The transportation of critically ill patients into or outside the hospital (ICU) has been associated with several adverse events ${ }^{[1,2]}$. Mostly, patients admitted to the Intensive Care Unit (ICU) are considered to be critically ill. ICU can provide the best possible care to the patients, including monitoring, multiple organ support, frequent clinical round, and dedicated staff members for each patient. However, specific situations occur when the patient has to be transported out of the ICU to the best of the patient's interest. The benefits attached to the purpose of the transportation outweigh the risks. This literature review aims to summarize timely interventions, minimum standards for transportation, transport protocols, and recommendations to reduce critically ill patients to the potential risk in the ICU. We aim to improve the quality of patient care, risk evaluation, minimizing preventable hazards, standardization of the protocols, homogeneity of the modalities involved in the patient's transport, and ultimately improving the patient's health care environment. Findings show that, a total of $1.7 \%$ of adverse events during transportation was identified. In this study, 3383 charts of completed transports were observed ${ }^{[6]}$. The incidence of adverse effects is quite variable, i.e., from $1.7 \%$ to $75.7 \%$, and in other studies, it is sometimes recorded as high as $80 \%^{[4]}$.
\end{abstract}

Key Words: Transport, Critically Ill Patients, Early Interventions, Protocols, Recommendations

\section{INTRODUCTION}

Transport to and from ICU may fall under two main headings, i.e., intra-hospital transport (IHT) and inter-hospital transport. Intra-hospital transport (IHT) is a patient's movement within the hospital premises ${ }^{[7]}$ ${ }^{[8]}$. Inter-hospital transport is a patient's movement between two different medical centers ${ }^{[9]}$. The reason for these transports occurring in the hospital settings is to seek advanced medical care, facilitated by improved medical diagnostic procedures, imaging like computed tomography scan (CT scan), magnetic resonance imaging (MRI), nuclear medicine imaging, and gastrointestinal endoscopy. Anesthesiologists can play a crucial role in transportation by preventing, avoiding, or alleviating adverse events ${ }^{[3]}$.

Nevertheless, transportation can harm the patient's health. It has long been observed that such a procedure poses a potential risk of hypoxia, organ injury, hypotension or hypertension, ventilation problems, vascular access problems, limb immobilization, and airway problems, resulting in increased patient mortality morbidity ${ }^{[4]}$. Despite a vast literature on the hazards related to transportation, it has not yet been prevented or minimized to zero percent ${ }^{[5]}$. 


\section{Reasons for transportation:}

Transport for diagnostic reasons includes imaging examinations in the radiology department, hemodynamic and diagnostic endoscopic procedures in the endoscopic room. In comparison, therapeutic reasons include surgical interventions in the surgery department, endovascular procedures in the laboratory or cardiology department, and endoscopic interventions in the endoscopic room [11]. Moreover, the transport type may be an emergency or elective ${ }^{[13]}$.

It is reported in the study by Jia et al., that the most common reasons for intrahospital transport are for computed tomography imaging $(86.2 \%)$, followed by ultrasonography $(4.1 \%)$, radiations $(1.8 \%)$, MRI (1.6\%), endoscopy, and angiography $(0.9 \% \text { each })^{[13]}$.

\section{Risks and benefits of transport:}

The patient's transportation is aim to seek the following benefits; 1) to access and obtain the newest treatment (2) to refer the patient to a facility skilled at providing particular care (3) for re-evaluation or bringing changes in the treatment plan (4) for ensuring the provision of the best possible care. On the other hand, risks associated with transportation can be in the form of 1) complications occurring during transportation (2) possibility of information loss at receiving end as new health care team may take a while to get acquainted (3) interruption in the on-going health care at the backend during transfer out and again transfer back into ICU from a particular facility (4) personal reasons like anxiety about the new place and being distant from family ${ }^{[14]}$.

Thus, the anticipatory benefit for the patients in terms of improved survival and quality of health must always exceed the health risks associated with precluding transportation.

\section{Impact on the patients:}

Transporting patients is hazardous because it can cause physiological alterations via various mechanisms. The patient's movement can result in acceleration, deceleration, frequent changes in the posture, and change of surfaces. These variables are responsible for causing hemodynamic, respiratory, psychological, and neurological system complications. Furthermore, shifting from a protective environment of ICU and highly specialised equipment support to get treatment in a noisy place (hallways and elevators), having limited space in the vehicle for caregivers, issues of low light, bearing hard examining surfaces, and discomfort of the procedure itself are all the variables which can bring unwanted physiological changes. Thus, negatively impacting patient's health ${ }^{[6,15-19]}$. The transported patients are already suffering from some complications associated with multiple system disorders. Therefore, physiological impacts on critically ill patients during mobility can add insult to the injury if transportation does not occur smoothly ${ }^{[20]}$.

\section{Risk factors for Adverse Events:}

Medical literature has exclusively documented the variables that lead to adverse events during either intra-hospital or inter-hospital transport. The variables are identified as a multidisciplinary team, equipment, and patient itself. There are many essential types of equipment that should be supplied in the ICU. A list of required equipment types has been updated and published by Brighton and Sussex University Hospitals NHS Trust ${ }^{[21]}$.

Table 1 and Table 2 lists the critical risk factors of adverse events and potential problems that may occur after a thorough review of various crucial studies $[10,13,15,22$ 29]. 
Sibghatullah M Khan et.al. Transport of critically ill patients - a review of early interventions, protocols, and recommendations.

Table 1: lists the critical risk factors of adverse events

\begin{tabular}{|c|c|}
\hline System based risk factors & uman-based risk factors \\
\hline $\begin{array}{l}\text { Technical/Equipment related } \\
\text { - } \quad \text { Ventilated patient } \\
\text { - } \quad \text { Electrical or Gas failure } \\
\text { Workpgen depletion } \\
\text { - } \quad \text { Failure to follow a protocol } \\
\text { - } \quad \text { Inexperienced or new staff } \\
\text { Environment-related } \\
\text { - Not enough working space } \\
\text { - } \quad \text { Loud noises at working place } \\
\text { - } \quad \text { Vibrations at working place } \\
\text { Transport organization related } \\
\text { - } \quad \text { Emergency transport } \\
\text { - } \quad \text { Duration of transport } \\
\text { Treatment modification } \\
\text { transport for } \\
\text { Lack of coordination }\end{array}$ & $\begin{array}{l}\text { Patient-related } \\
\text { - } \quad \text { High Acute Physiology and Chronic Health Evaluation [APACHE II] score >20 } \\
\text { - } \quad \text { Poor Glasgow Coma Scale Score (PGCSS) } \\
\text { - } \quad \text { Poor Therapeutic Intervention Scoring System Score (PTISSS) } \\
\text { - } \quad \text { Pontinuous catecholamine support } \\
\text { - } \quad \text { Deranged Arterial Blood Gas (ABGs) before transport excluding bicarbonate } \\
\text { - } \quad \text { High lactate level }>2 \mathrm{mmol} / \mathrm{L} \\
\quad \text { Abnormal vital signs (particularly heart rate, respiratory rate, and saturation of inspired } \\
\text { oxygen) } \\
\text { - } \quad \text { Sow Glucose level } \\
\text { - } \quad \text { Patients suffering from head injuries } \\
\text { Knowledge-based errors } \\
\text { - } \quad \text { Error in problem recognition } \\
\text { Rule-based } \\
\text { - Inadequate patient preparation } \\
\text { - Inadequate sedation or analgesia } \\
\text { - Misusing equipment } \\
\text { Skill-based } \\
\text { - } \quad \text { Urgency or haste } \\
\text { - } \quad \text { Distraction or inattention } \\
\quad \text { Stress }\end{array}$ \\
\hline
\end{tabular}

- Stress

\begin{tabular}{|c|c|}
\hline System based risk factors & Human-based risk factors \\
\hline $\begin{array}{l}\text { Technical/Equipment related } \\
\text { - Failure of equipment (including power-related problems) } \\
\text { Workplace related } \\
\text { - } \quad \text { Communication gap } \\
\text { - Lack of protocols } \\
\text { - Lack of training } \\
\text { Transport related } \\
\text { - Multiple times transportation of the same patient } \\
\text { - Fluid change for transport }\end{array}$ & \begin{tabular}{l}
\multicolumn{2}{l}{ Rule-based } \\
- $\quad$ Inadequate patient preparation \\
- $\quad$ Incorrect patient assessment \\
- $\quad$ Failure to check equipment
\end{tabular} \\
\hline
\end{tabular}

Different authors have identified significant risk factors collected and categorized under two main headings in this document; 1) System based risk factors (2) Human-based risk factors. Thus, several risk factors impact the patient's health and result in adverse events.

\section{Types of Adverse Events:}

The types of adverse events occurring during transportation are broadly classified under two main headings; (1) patient-related adverse events, equipment-related adverse events. Some authors have classified complications based on the human body's systems (cardiovascular complications, respiratory complications, and other vital signs). Nevertheless, the second classification is considered to be a part of patient-related adverse events.

Details of classifications of adverse events are given in Tables 3 and Table 4, while unclassified adverse effects are listed in table $5^{[7,10,25,27,30-38]}$.

Table 3: Equipment related adverse event

Equipment related adverse event

Adverse events related to ventilation

- $\quad$ Failure of connection

- Leakage from bag

- Interruption in Oxygen supply due to reduced reserve in the cylinder

Adverse events related to monitoring

- Interference in the track record

- Malfunctioning of equipment

- Inability to see the display of equipment

- Interruption in the arterial line

Adverse events related to an intravenous line

- Difficult access

- Accidental removal of the catheter

- $\quad$ Short IV lines

Adverse events related to the vehicle's equipment

- $\quad$ Poor display of monitors

- Lack of adequate suction

- Crowding of staff

- Improper access to patient

Adverse events related to an infusion pump

- Inadequate medication supply

- $\quad$ Failure of battery

- Increased vasopressor dose

Adverse events related to intravenous poles

- Inability to push during transport 
Sibghatullah M Khan et.al. Transport of critically ill patients - a review of early interventions, protocols, and recommendations.

Table 4: Patient-related adverse events

\begin{tabular}{|c|c|c|}
\hline \multicolumn{3}{|l|}{ Patient-related adverse events } \\
\hline \begin{tabular}{ll}
\multicolumn{2}{l}{ Cardiovascular complications } \\
- & Hypertension \\
- & Tachycardia \\
- & Hypotension \\
- & Bradycardia \\
- & Bleeding \\
- & Arrhythmias \\
- & ECG changes \\
- & Cardiac arrest \\
Metabolic/Acid-Base Complications \\
- & Metabolic acidosis \\
- & Metabolic alkalosis \\
- & Respiratory acidosis \\
- & Respiratory alkalosis
\end{tabular} & $\begin{array}{ll}\text { Respiratory complications } \\
\text { - } & \text { Oxygen desaturation } \\
\text { - } & \text { Increased pressures in the airway } \\
\text { - } & \text { Blockage of airways secondary to secretions } \\
\text { - } & \text { Loss of chest tube } \\
\text { - } & \text { Excessive cough } \\
\text { - } & \text { Extubation } \\
\text { - } & \text { Pneumothorax } \\
\text { - } & \text { Atelectasis } \\
\text { - } & \text { Pulmonary embolism } \\
\text { - } & \text { Bronchospasm }\end{array}$ & $\begin{array}{l}\text { Endocrine Complications } \\
\text { - } \quad \text { Hyperglycemia } \\
\text { - } \quad \text { Hypoglycemia } \\
\text { CNS complications } \\
\text { - } \quad \text { Raised intracranial pressure } \\
\text { - } \quad \text { Agitation } \\
\text { - } \quad \text { Vomiting } \\
\text { - } \quad \text { Pain or discomfort } \\
\text { - } \quad \text { Spinal cord traction } \\
\text { - } \quad \text { Cervical spine injury } \\
\text { - } \quad \text { Exacerbation of the existing trauma } \\
\text { Infection } \\
\text { - } \quad \text { Ventilator-associated pneumonia (VAP) } \\
\text { - } \quad \text { Exposure to other infections }\end{array}$ \\
\hline
\end{tabular}

Some other unforeseen events are not classified in this article. They are given in the following table:

Table 5: Unclassified adverse events

\begin{tabular}{|l|l|}
\hline \multicolumn{2}{|l|}{ Miscellaneous } \\
\hline - & Moving to the wrong location/direction \\
- & Lack of communication between sending and receiving \\
- & Leaving without enough/appropriate drugs \\
- & Delayed receiving of the patient \\
- & Obstacles in the pathway or elevators \\
- & Shifting on inappropriate bed \\
\hline
\end{tabular}

According to several studies, most of the adverse effects were equipment-related adverse rather than patient-related ${ }^{[22,28,34]}$. In a cohort study, data analysis of approximately 293 critically ill patients (according to the World Health Organization classification of patients) was performed. It was observed that only $23.5 \%$ of the adverse events were associated with equipment failure, while $44.1 \%$ of adverse events were inherent to the patient in the form of physiological alterations ${ }^{[39]}$. As observed in table 3, equipment-related adverse events are further divided into "Adverse Events" due to ventilation, monitoring, intravenous line, procedure room, infusion pump, and intravenous pole mismanagement (mismanagement based on lack of practice, foreign technology, and limited training) ${ }^{[40]}$.

Concerning the equipment issues, ventilation problems, monitoring negligence, battery failure, and disruption in the oxygen supply are described to occur most frequently (percentages?).
On the other hand, patient-related events are divided based on systems like the cardiovascular system, respiratory system, central nervous system (CNS), infections, endocrine system, and metabolic acid-base disorder. In this category, common complications belong to the cardiovascular system and respiratory system. Another critical group of adverse events has been listed in table $5^{[11]}$. They are not categorised under the main headings but include events arising from delays and lack of teamwork.

\section{Guidelines to conduct patient transportation:}

The success of transporting patients lies in making the whole process efficient and organised. To conduct successful transportation without complications, practice guidelines have been formulated by Warren et al., compiled from various prospective studies, retrospective reviews, anecdotal reports, and consensus opinions. The author proposes that there must be a formulated plan in each hospital for conducting inter-hospital or intra-hospital transport, which should comply with the proper standard operating procedures (SOP's) ${ }^{[5]}$. The principal source of these SOP's or practice guidelines are; the Society of Critical Care Medicine (SCCM) guidelines published in 1993 and revised recently in 2016, Intensive Care Society (ICS) guidelines initially published in 1992 and updated in 2002, and finally the Australian and New Zealand College of Anesthetists (ANZCA) minimum standards published in 2003 and revised in $2017^{[17,41-}$ 
43] [44]. All these documents present almost the same recommendations. The core components are as follows;

- Pre-transport coordination and communication: whenever the patient is transported from one location to another, it is essential to maintain patient care quality throughout the process. The recipient team should be conveyed the patient-specific requirement to ensure the safe transportation of the patient. Availability of resources is considered to be an essential prerequisite for patient safety.

- Accompanying personnel: authors recommend the presence of two staff members in case of transportation. Two scenarios depending upon the patient's conditions are considered; 1) Stable patient, non-intubated/ventilated- one accompanying person is a nurse trained in critical care while the second accompanying person may be a respiratory therapist/registered nurse/critical care technician as per needs

2) Unstable patient, intubated/ventilated patient - one accompanying person is a nurse trained in critical care. Simultaneously, the second person must be a physician trained in airway management, advanced cardiac life support (ACLS), and critical care

- Accompanying equipment: there is a list of recommended minimum transport equipment and medications.

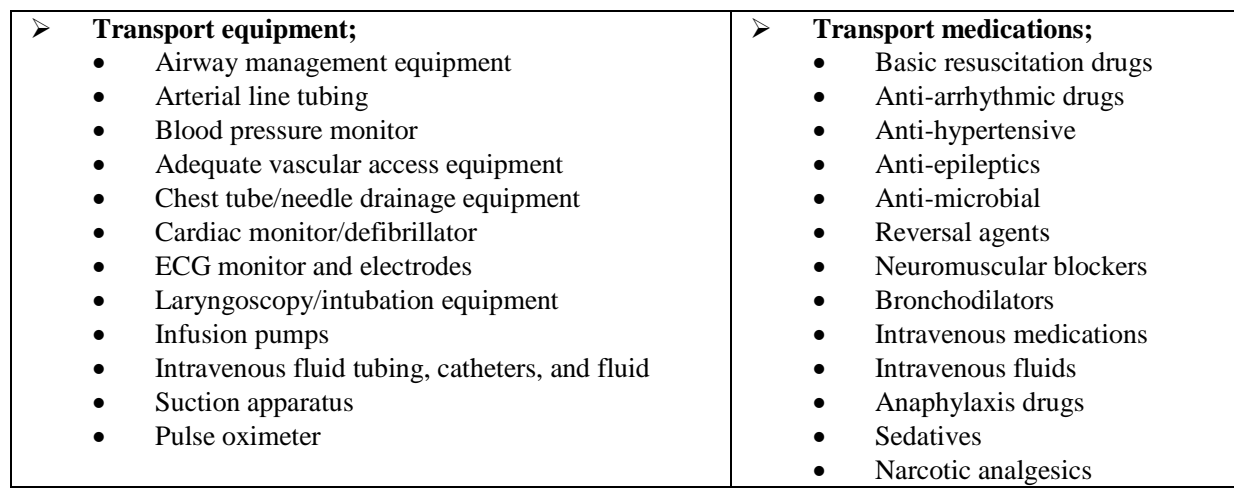

The different medication types and the minimum amount required to differ according to the distance to be travelled or the destination to be reached.

- Monitoring during transport: patients must be continuously monitored during their journey out of the ICU. It is essential to perform the monitoring of vital parameters with selected additional parameters. During transport, the monitoring list includes continuous electrocardiogram (ECG) readings, continuous pulse oximetry, periodic blood pressure (BP) measuring, pulse rate observation, and respiratory rate checkup. Additional monitoring may include capnography, intra-arterial blood pressure measuring, pulmonary artery pressure, and intracranial pressure measuring.

Capnography is the most crucial component in the transportation of patients on ventilators. It is also listed in the additional monitoring parameters for nonventilated patients. Moreover, capnography ranks only second to pulse oximetry in monitor-based-detection of adverse events like oesophageal intubation and circuit disconnection. Thus, monitoring with pulse oximetry, capnography, and airway pressures can detect most airway-associated adverse events ${ }^{[30,45]}$. If a combination of pulse oximetry, capnography, BP recording, and a fraction of inspired oxygen $(\mathrm{FiO} 2)$ is used during monitoring, it has the potential 
to detect up to $95 \%$ of incidents related to monitors ${ }^{[30]}$.

\section{Assessment of Patient:}

The study by Warren et al., has not just provided a review and summary of transportation guidelines. It has additionally added two more components to critically ill patient's medical care, and thus the existing protocols are being updated accordingly. The authors have taken into account two assessment approaches while transporting patients. One of them being the "head to toe" assessment, and the other one is the "The Airway, Breathing, Circulation, Disability, Exposure (A-B-C-D-E)" assessment approach $^{[12]}$.

Head-to-toe assessment of a patient in ICU is part of critical care nurse training and intensivist. It is also a documented protocol to assess the patients every time the shift changes occur in the ICU. Books of physical examination have also quoted it as an essential aspect of ICU care. A comprehensive assessment of the patient forms the basis of monitoring the changes and identifying the alterations to accurately understand the patient's health status. A list of possible adverse events is published by Dewhurst et al. ${ }^{[46]}$. This list has been adapted from the "Royal College of Anaesthetists' list of critical incidents."

The second assessment approach discussed by Warren et al., is the "A-B-CD-E" approach ${ }^{[12]}$. This approach is more practical and accurate in providing essential clues regarding patient conditions since it is a structured assessment paradigm. A structured assessment is based on fixed methods to assess the patients, grading the pain's severity and neurological complications [47]. Detailed and stepwise application of the "A-B-C-D-E" approach is available in the literature. However, more importantly, this approach provides lifesaving treatment, buying time for appropriate interventions, and assessments to formulate transportation tools. It also improves critical care while transporting patients, either intra-hospital or inter- hospital. These are crucial components in the context of the purpose of this manuscript $[48,49]$

\section{Transport tool:}

Using two assessment approaches to be incorporated into the existing transportation protocols by Warren et al., has formulated a comprehensive transportation tool ${ }^{[5]}$. It is a two-page document that constitutes the transport process's critical components, including; preparation, assessment, monitoring, and documentation. The tool comprises of 1) pre-transport checklist, (2) health assessment form, (3) checklist of destination, (4) chart of observation, (5) sections devoted to record complications, (6) reminder for checking equipment and oxygen before leaving.

It is an essential contribution towards critical care evaluation by the authors who have observed poor compliance with the use of the existing tools, including; limited use of resources by the ICU staff nurses, lack of teamwork between departments or communication between multidisciplinary teams, and lacking the engagement of the teams at the receiving department/hospital ${ }^{[12]}$. For this purpose, in the United States (US), a checklist (COBRA/EMTALA) is followed, up to the maximum possible limit ${ }^{[12]}$. An established and organized transport can pave the way for increased patient safety.

A similar form of transportation tool is formulated by Esmail et al., known as "Transport 43Decision Scorecard." It is a visual assessment tool that has been made by using "Plan-Do-Study-Act (PDSA)" cycles department of critical care medicine, Calgary health region ${ }^{[50]}$.

\section{Impact of protocols and transport tools} on patient care:

Procedural guidelines provide a framework to execute successful transportation. Simultaneously, transport tools, checklists, and other documents help keep records and adhere to the mandatory 
protocols to ensure a smooth transition of patients from one environment to another, maintaining physiological and clinical stability.

All these protocols help to achieve required critical care, and adherence to them can minimize the risks to the minimum levels. As reported in Choi et al., it has been observed that adverse effects were only reduced from $36 \%$ to $22 \%$ with the help of transportation checklists ${ }^{[51]}$. It has been observed that despite the presence of critical care guidelines, adverse events continue to occur in hospital settings during transportation. This could be due to the lack of compliance with the protocols to provide necessary care to the patients ${ }^{[52]}$. Ulrich Strauch and his colleagues have analysed the data from tertiary care hospitals of South East Netherland. They have reported that no negative impact on the patient's health was observed during the 344 analysed data sets during interhospital transportation. The transportation was carried out by a team dedicated to transport and local mobile intensive care unit ${ }^{[23]}$. In 2017, they also identified the lacking of evidence-based criteria to regulate transportation quality ${ }^{[53]}$.

\section{Role of "A-B-C-D-E" approach is early intervention:}

Adverse events tend to occur despite the presence of comprehensive guidelines and transport tools. Thus, impacting the "first line of defense." However, sometimes it is inevitable to avoid these adverse events. There arises the need for a "second line of defense." Here comes the role of early interventions and accurate assessments. Australian Incident Monitoring Survey (AIMS) reports that more than $80 \%$ of the errors occur due to human mistakes ${ }^{[30]}$. Thus, from another perspective, using the "A-B-C-D-E" approach can be acquired as an early intervention to control the deterioration of the patient's health. The importance of the "A-B-C-D-E" approach as early intervention can also be estimated because it involves airways, breathing, and circulation. Hence, it covers commonly occurring complications, which are mostly related to the respiratory system and cardiovascular system. This single approach can prevent secondary brain injury due to prolonged hypoxia and hypoperfusion ${ }^{[30]}$. Furthermore, this approach is used as an early intervention, which does not require a definite diagnosis. It can provide immediate treatment for life-threatening signs and buy critical time until the patient is shifted to an appropriate critical care facility ${ }^{[47-49]}$.

\section{Recommendations:}

A comprehensive review of the guidelines regarding the transportation of critically ill patients has already been presented. All the guidelines can be considered as recommended steps to be conducted as an inter-hospital or intrahospital transportation protocol with the minimum chances for adverse events to occur. However, an additional list of recommendations can boost the protocol's effectiveness, conveys information present in the set of guidelines to refine critical care, promotes the welfare of patient's health, and intensifies the care provided by intensivists and other staff members. Key recommendations extracted from the literature have been grouped under the respective headings in this document, which are not yet been discussed before in any other literature. The following recommendations are considered to be pivotal;

Patient related recommendations ${ }^{[15,16,27]}$ :

- It is crucial to stabilise the patient near to the normal physiological state as much as possible before executing the transportation

- A quick risk-benefit analysis must be carried out after stabilising before finalising the decision to transport

- Patients must be appropriately sedated or even curarized. 
Staff related recommendations $[8,10,11,15$, 18, 29, 54, 55]:

- The anticipation of unexpected events must always be done

- A specialised and experienced team must escort critically ill patients

- Several specialized training courses must be made mandatory for ICU staff

- Simulation training would help to validate the competency of the transport team

- The hospitals and teams may participate in workshops and training, for example, "Healthcare Failure Mode and Effects Analysis (HFMEA)" and "Medical Transportation Program (MTP)"

A brief discussion on HFMEA is essential here. As identified in the study of Lin et al., it has been observed that using an improvement program for a reminderassisted briefing during transportation is quite crucial in controlling adverse events. A team leader (clinician) ensures the "Mnemonics" assigned to each escort team member are followed. The "Mnemonics" are as follows ${ }^{[11][56]}$;

1. VITAL for ICU Nurse - Vital signs, Infusions, Tubes, Alarms and Leave

2. STOP for Respiratory therapist Secretions, Tubes, Oxygen, and Power

3. STOP for Radiology technician - Speak out, Tubes, Other, and Position

Results have shown that the implementation of this program has significantly reduced adverse events ${ }^{[11]}$

Transport related recommendations ${ }^{[20,28,}$ 52]:

- The decision to transport patients must also be taken after considering the destination of the receiving facility, cost of medical care, and health capability of the patient to bear the transportation

- Checklists must be easy to fill and easy to understand because protocols are too vague and exhausting

- For inter-hospital transport, it is recommended to use the "Mobile
Intensive Care Unit (MICU)" rather than a standard ambulance

Monitoring related recommendations ${ }^{[10,}$ 12,13,15, 16, 54, 57, 58]:

- Monitoring of end-tidal carbon dioxide $\left(\mathrm{CO}_{2}\right)$ and capnograms must be essential in the transportation in ventilated patients.

- Reliance on mechanical ventilation rather than manual ventilation is better due to the superiority of the former ventilator. It is recommended to avoid using manual ventilation and only use it in emergency settings.

- The exact function and capacity of the portable ventilator must be known to the team (primary ventilator, intermediate ventilator, or high-performance ventilator)

- A system for tracking, analysing, and evaluating the patient health status must be considered in the future. It is recommended to create an intra-hospital transport-related monitoring database

\section{Research related recommendations:}

- Regular evaluation or studies should be conducted to identify the gaps in critical care practice

- Checklists with the scoring system should be formulated

\section{DISCUSSION}

Intra-hospital or inter-hospital transport is the fundamental component for critically ill patient's management as it serves two primary purposes. Firstly, it aids in the diagnosis. Secondly, it aids in therapeutic intervention. Simultaneously, the third purpose of transport may be "revision," which specifies a particular need for second surgery ${ }^{[10]}$. Diagnostic purposes $(70 \%)$ are more significant than therapeutic purposes $(15 \%)$ in most of the cases, while $15 \%$ of the transports occur for "revision" (supplemental surgical procedure) ${ }^{[10]}{ }^{[11]}$. The medical care which underlies the concept of patient transportation is advanced level or higher intensity therapy 
consisting of technical, cognitive, and procedural care, which is beyond the scope of former ICU or the hospital ${ }^{[12]}$.

\section{CONCLUSION}

An in-depth review of the literature reveals that adverse events spring from equipment failures and human errors. The latter cause is vital to discuss, and it is crucial to improve it because controlling the human factor requires a great deal of education, coaching, time investment, and energy. It is a process that takes time to evolve within the local system and working environment of the hospital and, ultimately, the whole healthcare system. In the meantime, efforts must be made in quick checklists for accurate assessments, mnemonics for prompting the first line of defense against potential adverse events and using the "A-B-C-D-E" approach as a second line of defense.

\section{Acknowledgement: None}

\section{Conflict of Interest: None}

\section{Source of Funding: None}

\section{REFERENCES}

1. Alamanou, D.G. and H. Brokalaki, Intrahospital transport policies: The contribution of the nurse. Health Science Journal, 2014. 8(2): p. 166.

2. Flabouris, A., Patient referral and transportation to a regional tertiary ICU: patient demographics, severity of illness and outcome comparison with non-transported patients. Anaesthesia and intensive care, 1999. 27(4): p. 385.

3. Wacker, J. and S. Staender, The role of the anesthesiologist in perioperative patient safety. Current opinion in anaesthesiology, 2014. 27(6): p. 649-656.

4. Flabouris, A., W. Runciman, and B. Levings, Incidents during out-of-hospital patient transportation. Anaesthesia and intensive care, 2006. 34(2): p. 228-236.

5. MacDonald, R.D., B.A. Banks, and M. Morrison, Epidemiology of adverse events in air medical transport. Academic emergency medicine, 2008. 15(10): p. 923931.

6. Kue, R., et al., Adverse clinical events during intrahospital transport by a specialized team: a preliminary report. American Journal of Critical Care, 2011. 20(2): p. 153-162.

7. Knight, P.H., et al., Complications during intrahospital transport of critically ill patients: Focus on risk identification and prevention. International journal of critical illness and injury science, 2015. 5(4): p. 256.

8. Lin, S.-J., et al., Improving patient safety during intrahospital transportation of mechanically ventilated patients with critical illness. BMJ open quality, 2020. 9(2): p. e000698.

9. Shojania, K.G., et al., Making health care safer: a critical analysis of patient safety practices. Evid Rep Technol Assess (Summ), 2001. 43(1): p. 668.

10. Lahner, D., et al., Incidence of complications in intrahospital transport of critically ill patients-experience in an Austrian university hospital. Wiener Klinische Wochenschrift, 2007. 119(13-14): p. 412-416.

11. Gimenez, F.M.P., et al., Analysis of adverse events during intrahospital transportation of critically ill patients. Critical care research and practice, 2017. 2017.

12. Warren, J., et al., Guidelines for the interand intrahospital transport of critically ill patients. Critical care medicine, 2004. 32(1): p. 256-262.

13. Jia, L., et al., High incidence of adverse events during intra-hospital transport of critically ill patients and new related risk factors: a prospective, multicenter study in China. Critical Care, 2016. 20(1): p. 12.

14. Iwashyna, T.J., The incomplete infrastructure for interhospital patient transfer. Critical care medicine, 2012. 40(8): p. 2470-2478.

15. Fanara, B., et al., Recommendations for the intra-hospital transport of critically ill patients. Critical Care, 2010. 14(3): p. R87.

16. Blakeman, T.C. and R.D. Branson, Interand intra-hospital transport of the critically Ill Discussion. Respiratory care, 2013. 58(6): p. 1008-1023.

17. Australasian College for Emergency Medicine, A., N.Z.C.o. Anaesthetists, and J.F.o.I.C. Medicine, Minimum standards for 
intrahospital transport of critically ill patients. Emergency Medicine, 2003. 15(2): p. 202-204.

18. Gillman, L., et al., Adverse events experienced while transferring the critically ill patient from the emergency department to the intensive care unit. Emergency medicine journal, 2006. 23(11): p. 858-861.

19. Stevenson, V.W., C. Haas, and W.L. Wahl, Intrahospital transport of the adult mechanically ventilated patient. Respiratory care clinics of North America, 2002. 8(1): p. 1-36.

20. Wiegersma, J.S., et al., Quality of interhospital transport of the critically ill: impact of a Mobile Intensive Care Unit with a specialized retrieval team. Critical care, 2011. 15(1): p. R75.

21. Trust, B.a.S.U.H.N. Equipment, devices and procedures in the Intensive Care Unit. 2020 01.01.2020 [cited 2018 01.01.2018]; Available from: https://www.bsuh.nhs.uk/wpcontent/uploads/sites/5/2016/09/Equipmentdevices-and-procedures-in-the-IntensiveCare-Unit.pdf.

22. Parmentier-Decrucq, E., et al., Adverse events during intrahospital transport of critically ill patients: incidence and risk factors. Annals of intensive care, 2013. 3(1): p. 10.

23. Strauch, U., et al., Short-term outcomes and mortality after interhospital intensive care transportation: an observational prospective cohort study of 368 consecutive transports with a mobile intensive care unit. BMJ open, 2015. 5(4).

24. Tang, Y., et al., Clinical predictors of adverse outcome in severe sepsis patients with lactate 2-4 $\mathrm{mM}$ admitted to the hospital. QJM: An International Journal of Medicine, 2015. 108(4): p. 279-287.

25. Zhang, Z. and $X$. Xu, Lactate clearance is a useful biomarker for the prediction of allcause mortality in critically ill patients: a systematic review and meta-analysis. Critical care medicine, 2014. 42(9): p. 21182125.

26. Bercault, N., et al., Intrahospital transport of critically ill ventilated patients: a risk factor for ventilator-associated pneumonia-a matched cohort study. Critical care medicine, 2005. 33(11): p. 2471-2478.

27. Damm, C., et al. Complications during the intrahospital transport in critically ill patients. in Annales Francaises d'anesthesie et de Reanimation. 2005.

28. Papson, J.P., K.L. Russell, and D.M. Taylor, Unexpected events during the intrahospital transport of critically ill patients. Academic Emergency Medicine, 2007. 14(6): p. 574577.

29. Beckmann, U., et al., Incidents relating to the intra-hospital transfer of critically ill patients. Intensive care medicine, 2004. 30(8): p. 1579-1585.

30. Lovell, M., M. Mudaliar, and P. Klineberg, Intrahospital transport of critically ill patients: complications and difficulties. Anaesthesia and intensive care, 2001. 29(4): p. 400-405.

31. Schwebel, C., et al., Safety of intrahospital transport in ventilated critically ill patients: a multicenter cohort study. Critical care medicine, 2013. 41(8): p. 1919-1928.

32. de Lassence, A., et al., Pneumothorax in the intensive care unit: incidence, risk factors, and outcome. The Journal of the American Society of Anesthesiologists, 2006. 104(1): p. 5-13.

33. Orgeas, M.G., et al., Impact of adverse events on outcomes in intensive care unit patients. Critical care medicine, 2008. 36(7): p. 2041-2047.

34. Venkategowda, P.M., et al., Unexpected events occurring during the intra-hospital transport of critically ill ICU patients. Indian journal of critical care medicine : peer-reviewed, official publication of Indian Society of Critical Care Medicine, 2014. 18(6): p. 354-357.

35. Vincent, J.-L., et al., International Study of the Prevalence and Outcomes of Infection in Intensive Care Units. JAMA, 2009. 302(21): p. 2323-2329.

36. Conrad, B.P., et al., Eliminating log rolling as a spine trauma order. Surgical neurology international, 2012. 3(Suppl 3): p. S188S197.

37. Picetti, E., et al., Intra-hospital transport of brain-injured patients: a prospective, observational study. Neurocrit Care, 2013. 18(3): p. 298-304.

38. Zuchelo, L.T.S. and P.A. Chiavone, Intrahospital transport of patients on invasive ventilation: cardiorespiratory repercussions and adverse events. Jornal Brasileiro de Pneumologia, 2009. 35(4): p. 367-374. 
39. Gimenez, F.M.P., et al., Analysis of Adverse Events during Intrahospital Transportation of Critically Ill Patients. Critical Care Research and Practice, 2017. 2017: p. 6847124.

40. Morrow, M.S., Quality and Safety of Intermittent Intravenous Infusions. 2018, Case Western Reserve University.

41. Artide, S., Guidelines for the transfer of critically ill patients. Critical Care Medicine, 1993.

42. Jarden, R.J. and S. Quirke, Improving safety and documentation in intrahospital transport: development of an intrahospital transport tool for critically ill patients. Intensive and Critical Care Nursing, 2010. 26(2): p. 101-107.

43. McClave, S.A., et al., Guidelines for the provision and assessment of nutrition support therapy in the adult critically ill patient: Society of Critical Care Medicine (SCCM) and American Society for Parenteral and Enteral Nutrition (ASPEN). Journal of Parenteral and Enteral Nutrition, 2016. 40(2): p. 159-211.

44. Kolawole, H., et al., Australian and New Zealand anaesthetic allergy group/Australian and New Zealand College of anaesthetists perioperative anaphylaxis management guidelines. Anaesthesia and Intensive Care, 2017. 45(2): p. 151-158.

45. Williamson, J., et al., The capnograph: applications and limitations - an analysis of 2000 incident reports. Anaesthesia and intensive care, 1993. 21(5): p. 551-557.

46. Dewhurst, A., et al., Medical repatriation via fixed- wing air ambulance: a review of patient characteristics and adverse events. Anaesthesia, 2001. 56(9): p. 882-887.

47. Munroe, B., et al., The impact structured patient assessment frameworks have on patient care: an integrative review. Journal of Clinical Nursing, 2013. 22(21-22): p. 2991-3005.

48. Thim, T., et al., ABCDE--a systematic approach to critically ill patients. Ugeskrift for laeger, 2010. 172(47): p. 3264-3266.

49. Thim, T., et al., Initial assessment and treatment with the Airway, Breathing, Circulation, Disability, Exposure (ABCDE) approach. International journal of general medicine, 2012. 5: p. 117.
50. Esmail, R., et al., Is your patient ready for transport? Developing an ICU patient transport decision scorecard. Healthc Q, 2006. 9(special number): p. 80-86.

51. Choi, H.K., et al., A before-and afterintervention trial for reducing unexpected events during the intrahospital transport of emergency patients. The American journal of emergency medicine, 2012. 30(8): p. 1433-1440.

52. Shirley, P.J. and J.F. Bion, Intra-hospital transport of critically ill patients: minimising risk. 2004, Springer.

53. Strauch, U., et al., QUIT EMR trial: a prospective, observational, multicentre study to evaluate quality and 24 hours posttransport morbidity of interhospital transportation of critically ill patients: study protocol. BMJ open, 2017. 7(3).

54. Quenot, J.-P., et al., Intrahospital transport of critically ill patients (excluding newborns) recommendations of the Société de Réanimation de Langue Française (SRLF), the Société Française d'Anesthésie et de Réanimation (SFAR), and the Société Française de Médecine d'Urgence (SFMU). Annals of intensive care, 2012. 2(1): p. 1-6.

55. Droogh, J.M., et al., Inter-hospital transport of critically ill patients; expect surprises. Critical care, 2012. 16(1): p. R26.

56. Bergman, L., et al., Development and initial psychometric testing of the Intrahospital Transport Safety Scale in intensive care. BMJ open, 2020. 10(10): p. e038424.

57. Nakamura, T., et al., Intrahospital transport of critically ill patients using ventilator with patient-triggering function. Chest, 2003. 123(1): p. 159-164.

58. Kluge, S., H.J. Baumann, and G. Kreymann, Intrahospital transport of a patient with acute exacerbation of chronic obstructive pulmonary disease under noninvasive ventilation. Intensive care medicine, 2005. 31(6): p. 886-886.

How to cite this article: Khan SM, Lance MD, Mariam Ali Karrar Elobied. Transport of critically ill patients - a review of early interventions, protocols, and recommendations. Int J Health Sci Res. 2021; 11(4): 133-143. DOI: https://doi.org/10.52403/ijhsr.20210418 